\title{
Multiply charged metal cluster anions
}

\author{
Constantine Yannouleas, ${ }^{1}$ Uzi Landman, ${ }^{1}$ Alexander Herlert, ${ }^{2}$ and Lutz Schweikhard ${ }^{2}$ \\ ${ }^{1}$ School of Physics, Georgia Institute of Technology, Atlanta, Georgia 30332-0430 \\ ${ }^{2}$ Institut für Physik, Johannes-Gutenberg Universität, D-55099 Mainz, Germany
}

(November 2000)

Formation, stability patterns, and decay channels of silver dianionic and gold trianionic clusters are investigated with Penning-trap experiments and a shell-correction method including shape deformations. The theoretical predictions pertaining to the appearance sizes and electronic shell effects are in remarkable agreement with the experiments. Decay of the multiply anionic clusters occurs predominantly by electron tunneling through a Coulomb barrier, rather than via fission, leading to appearance sizes unrelated to those of multiply cationic clusters.

Pacs Numbers: 36.40.Wa, 36.40.Qv, 36.40.Cg

Charging of macroscopic metal spheres is an old subject with scientific accounts dating back to Coulomb and Faraday [1]. Recently, electrical charging emerged as a central issue in connection with quantal nanostructures in diverse areas of condensed-matter, molecular, and cluster physics, such as artificially fabricated semiconductor nanodevices known as Quantum Dots [2], and gas-phase microsystems, such as fullerenes [3, t], large organic molecules [5], and metal microclusters [6 10].

In this Letter, we investigate the physical processes underlying generation of multiply anionic gas-phase metal clusters, $M_{N}^{Z-}$. The production and observation of gasphase multiply anionic aggregates had remained for many years a challenging experimental goal. This state of affairs changed in the last few years with the observation of several dianionic aggregates, including doubly negative fullerenes [3], metal-tetrahalide molecular dianions [11, and most recently dianionic gold clusters [12]. A few further observations of dianionic 13 and trianionic 114,15 gas-phase metal clusters have also been reported. In addition, we note that a few theoretical studies 16 of multiply charged anionic metal clusters have also appeared and that the earlier of them [16(a)] had anticipated several of the recent experimental developments; however, overall, the field of multiply anionic aggregates remains at its early stages.

Unlike the case of macroscopic metal spheres, where the number of positive or negative elementary charges that can be added is essentially arbitrary, with gasphase metal clusters this number is size-controlled due to quantum-confinement instabilities arising from the restriction of the excess charges to a very small space. In this Letter, we provide definitive evidence that multiply anionic metal clusters (irrespective of $Z$ ) behave in a way remarkably different from their cationic counter- parts. That is, they dissociate via a different decay channel, i.e., electron autodetachment through a repulsive Coulomb barrier (CB) 17], instead of fragmenting via fission. This results in appearance sizes unrelated to those known for the cationic species [6,8]. In addition, we show that the multiply charged metal-cluster anions exhibit electronic shell effects arising from magic major shells and subshells associated with ellipsoidal shapes [8,9], in full analogy with their neutral and cationic counterparts. We arrive at these conclusions through a careful and systematic comparison of theoretical predictions and original experimental observations for both doubly and triply anionic metal clusters. In particular, we investigate the stability and decay channels of $\mathrm{Ag}_{N}^{2-}$ and $\mathrm{Au}_{N}^{3-}$, and determine the corresponding appearance sizes $n_{a}^{Z-}$, where clusters with $N<n_{a}^{Z-}$ are energetically unstable.

Theoretically, two classes of decay channels need to be considered for the appearance sizes of the $\mathrm{M}_{N}^{Z-}$ clusters: (I) Binary (for $Z=2$ and $Z=3$ ) and ternary (for $Z=3$ ) fission channels,

$$
\begin{gathered}
M_{N}^{2-} \rightarrow M_{P}^{-}+M_{N-P}^{-}, \\
M_{N}^{3-} \rightarrow M_{P}^{2-}+M_{N-P}^{-}, \\
M_{N}^{3-} \rightarrow M_{P}^{-}+M_{Q}^{-}+M_{N-P-Q}^{-},
\end{gathered}
$$

which have well known analogs in the case of multiply cationic clusters [6 8] and atomic nuclei [18], and

(II) Electron autodetachment via tunneling through a repulsive Coulombic barrier [16],

$$
M_{N}^{Z-} \rightarrow M_{N}^{(Z-1)-}+e ; Z \geq 2,
$$

in analogy to proton and alpha decay in atomic nuclei 118, 19. For analysis of the energetics of these channels, we use a finite-temperature semi-empirical shellcorrection method (SCM), which incorporates triaxial shapes and which has been previously used successfully to describe the properties of neutral and cationic metal clusters [8,9]. The finite-temperature multiple electron affinities of a cluster of $N$ atoms of valence $v$ (we take $v=1$ for $\mathrm{Au}$ and $\mathrm{Ag}$ ) are defined as

$A_{Z}(N, \beta)=F(\beta, v N, v N+Z-1)-F(\beta, v N, v N+Z)$,

where $F$ is the free energy, $\beta=1 / k_{B} T$, and $Z \geq 1$ is the number of excess electrons in the cluster (e.g., the first, 


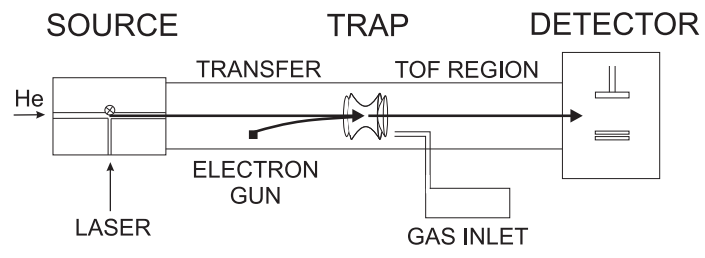

FIG. 1. Overview of the experimental setup.

second, and third electron affinities correspond to $Z=1$, $Z=2$, and $Z=3$ respectively). In the SCM, the free energy $F$ is separated into a smooth liquid-drop-model (LDM) part $\widetilde{F}_{\mathrm{LDM}}$ (varying monotonically with $N$ ), and a Strutinsky-type shell-correction term $\Delta F_{\mathrm{sp}}=F_{\mathrm{sp}}-\widetilde{F}_{\mathrm{sp}}$. $F_{\text {sp }}$ is the canonical (fixed $N$ at a given $T$ ) free energy of the valence electrons. The latter are treated as independent single particles moving in an effective mean-field potential (approximated by a modified Nilsson hamiltonian pertaining to triaxial cluster shapes). $\widetilde{F}_{\text {sp }}$ is the Strutinsky-averaged free energy. The smooth $\widetilde{F}_{\text {LDM }}$ contains volume, surface, and curvature contributions, whose coefficients and temperature dependencies are determined as described in Ref. [9]. In addition to the finite-temperature contribution due to the electronic entropy, the entropic contribution from thermal shape fluctuations is evaluated via a Boltzmann averaging [9(b)].

The smooth contribution $\widetilde{A}_{Z}(N, \beta)$ to the full multiple electron affinities $A_{Z}(N, \beta)$ can be approximated by the LDM expression $[16(\mathrm{a})]$,

$$
\widetilde{A}_{Z}=\widetilde{A}_{1}-\frac{(Z-1) e^{2}}{R(N)+\delta_{0}}=W-\frac{(Z-1+\gamma) e^{2}}{R(N)+\delta_{0}}
$$

where $R(N)=r_{s} N^{1 / 3}$ is the radius of the positive background $\left(r_{s}\right.$ is the Wigner-Seitz radius which depends weakly on $T$ due to volume dilation), $\gamma=5 / 8, \delta_{0}$ is an electron spillout parameter, and the work function $W$ is assumed to be temperature independent [we take $W(\mathrm{Au})=5.31 \mathrm{eV}$ and $W(\mathrm{Ag})=4.26 \mathrm{eV}]$.

The present experimental results have been achieved by use of a Penning trap system devoted to metal cluster research (Fig. 1) [20]: A Smalley-type ion source produces the clusters by laser vaporization of a metal wire in the presence of a helium gas pulse [21]. The resulting clusters are either neutral or singly charged. The anionic species are transferred by ion-optical elements through differential-pumping stages to a Penning trap where they are captured in flight [22]. The storage is provided by the combination of a $5 \mathrm{~T}$ homogeneous magnetic field and an electric trapping potential along the magnetic field lines [23]. In order to generate higher charge states, an electron beam of about $40 \mathrm{eV}$ is sent through the trap. Simultaneously supplied argon atoms are ionized producing low-energy secondary electrons which stay stored in the trap volume. Some of these electrons attach to the clusters, thus charging them up. This reaction is analyzed
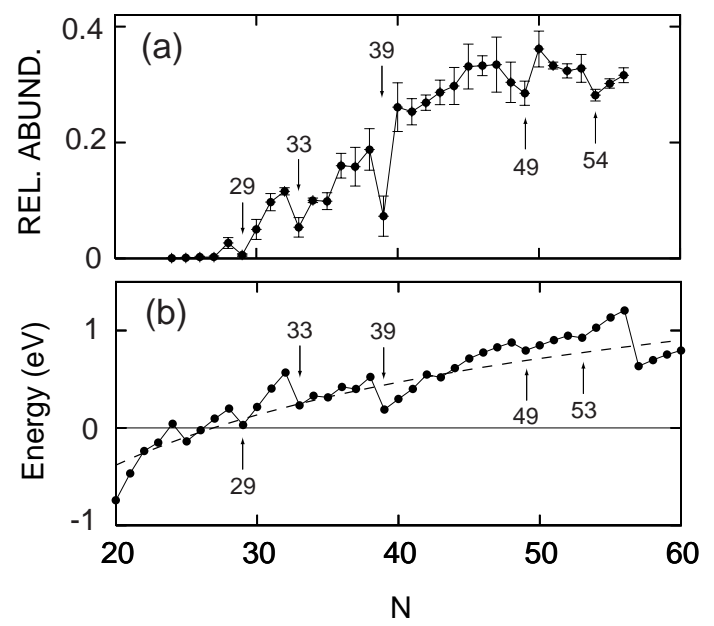

FIG. 2. (a) Experimental yields of dianionic silver clusters $\mathrm{Ag}_{N}^{2-}$, plotted versus cluster size. Most measurements have been repeated (some several times). The error bars indicate the statistical uncertainty or the deviations between different measurements (whatever is larger). (b) Theoretical SCM second electron affinities $A_{2}$ for $\mathrm{Ag}_{N}^{2-}$ clusters at $T=300 \mathrm{~K}$.

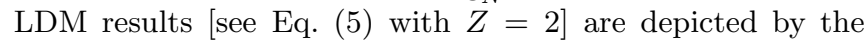
dashed line.

by ejection of the clusters from the trap and time-offlight mass spectrometry. Single-ion counting has to be performed, since only a few tens of ions are observed in each experimental cycle. The signal intensity of several hundred sequences is summed to increase the statistical significance of the data.

The conversion yield from mono- to di- and trianionic metal clusters is a complex function of many experimental parameters. The details of the process still await further elucidation. Nevertheless, when care is taken that the parameters are kept unchanged during an experimental sequence, valuable information on the clusters' properties can be gained from the relative conversion yields. In particular, based on the case of gold cluster dianions, it has been suggested $[13,16(\mathrm{~b})]$ that the resulting experimental yield pattern can be correlated to the clusters' multiple electron affinities. The cases of $\mathrm{Ag}_{N}^{2-}$ and $\mathrm{Au}_{N}^{3-}$ considered here show this to be a general property applicable to any excess charge and cluster species.

Fig. 2(a) displays the observed relative abundances of the dianionic silver clusters. Dianionic $\mathrm{Ag}_{N}^{2-}$ have been observed for $N \geq 24$. In general, the yield is very low for $N<28$ and then increases linearly up to about size $N=45$ where it levels off. Embedded in this general trend are pronounced dips at $N=29,33$, and 39, as well as around $N=49$ and 54 .

To gain further insight into these experimental trends, Fig. 2(b) presents the theoretical SCM results for the second electron affinity $A_{2}$ of silver clusters in the size range $20 \leq N \leq 60 . \quad N=24$ with $A_{2}=0.04 \mathrm{eV}$ forms a very weak island in front of the main stability 


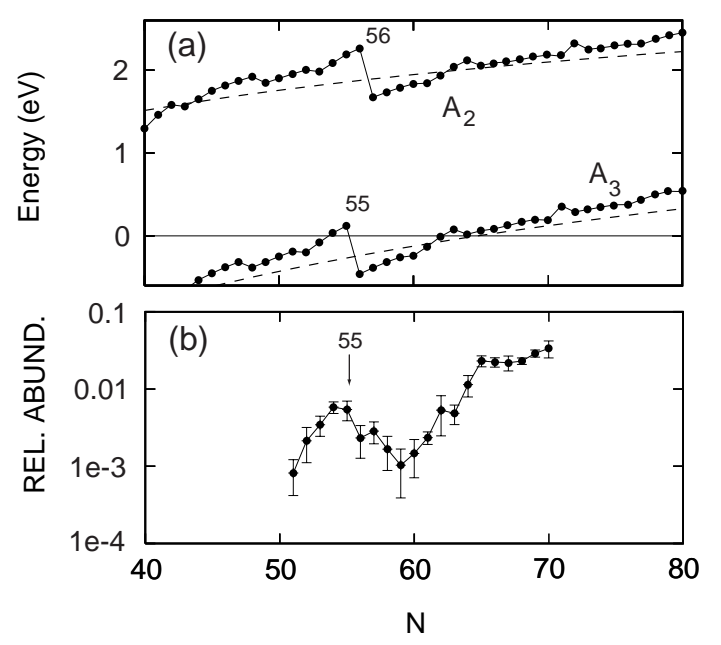

FIG. 3. (a) Calculated second ( $A_{2}$, upper curve) and third ( $A_{3}$, lower curve) electron affinities for $\mathrm{Au}_{N}$ clusters at $T=300 \mathrm{~K}$ in the size range $40 \leq N \leq 80$. Theoretical results from SCM calculations are connected by a solid line, and LDM results [see Eq. (5) with $Z=2$ and 3] are depicted by the dashed lines. (b) Experimental average relative abundances of $\mathrm{Au}_{N}^{3-}$ as compared to $\mathrm{Au}_{N}^{2-}$ clusters in the same size range (error bars as in Fig. 2). Notice the logarithmic scale.

branch starting at $N=27$. Disregarding this weak island, the appearance size for the main stability island of $\mathrm{Ag}_{N}^{2-}$ (i.e., the smallest size with $A_{2}>0$ ) is predicted to be $n_{a}^{2-}(\mathrm{Ag})=27$. The theoretical curve for the electron affinities and the experimental measurements correlate to a remarkable degree. In particular: (I) The smallest size seen in the experiment is $N=24$, but the yields from $N=24$ to 27 are very small; (II) At $N=27$, the experimental yields start on the average to increase sharply and keep increasing for $27 \leq N \leq 45$, in agreement with the theoretical prediction for $n_{a}^{2-}(\mathrm{Ag})$ and with the trend of the theoretical curve; and (III) The dips in the measured yields [marked by arrows in Fig. 2(a)] are in accordance with corresponding minima in the theoretical curve.

Underlying the theoretical and experimental patterns shown in Fig. 2 are electronic shell effects [compare in Fig. 2(b) the shell-corrected results indicated by the solid line with the LDM curve] combined with energy-lowering shape deformations for the open-shell clusters [9]. These deformations are akin to Jahn-Teller distortions and are associated with the lifting of spherical spectral degeneracies and the formation of electronic subshells (for 30, 34, and 50 electrons) in addition to the (magic) major shell (for 40 electrons).

Next we discuss the case of trianionic metal clusters. In Fig. 3(a), we display the SCM theoretical results [24,25] for the second, $A_{2}$, and third, $A_{3}$, electron affinities of $\mathrm{Au}_{N}$ clusters in the size range $40 \leq N \leq 80$. Multiply anionic clusters $\mathrm{M}_{N}^{Z-}$ with $A_{Z}<0$ are unstable [16] against electron emission via tunneling through a CB. In the case of doubly anionic gold clusters, all sizes in the

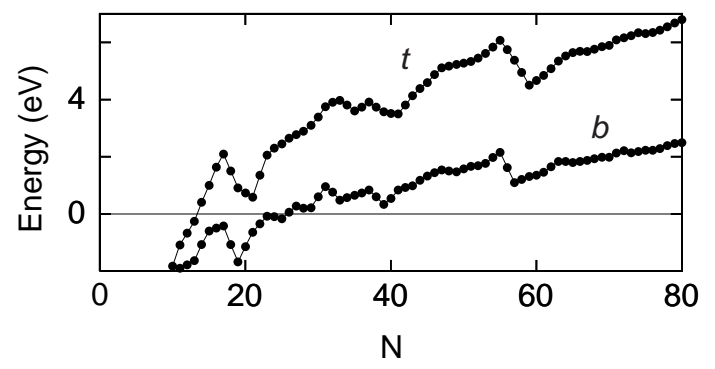

FIG. 4. Theoretical SCM fission dissociation energies for the most favorable channel $\left(\Delta_{N, \gamma}^{3-}\right.$ in $\left.\mathrm{eV}\right)$ at $T=300 \mathrm{~K}$ for binary $(\gamma=b$, lower curve) and ternary $(\gamma=t$, upper curve) fission of trianionic $\mathrm{Au}_{N}^{3-}$ clusters. Exothermic fission $\left(\Delta_{N, \gamma}^{3-}<0\right)$ is found only for the smallest clusters with $N \leq 25$.

aforementioned range are stable, i.e., they have $A_{2}>0$ [see upper curve in Fig. 3(a)]. In contrast, for the goldcluster trianions, those with $N \leq 53$ and $56 \leq N \leq 62$ have $A_{3}<0$. Thus, the appearance size for $\mathrm{Au}_{N}^{3-}$ is $n_{a}^{3-} \approx 54$. Apparently, the major shell closure at 58 electrons (corresponding to the $\mathrm{Au}_{56}^{2-}$ parent of the triply charged $\mathrm{Au}_{56}^{3-}$ cluster) creates an island of stability (the clusters with $N=54$ and 55 have $A_{3}>0$ ) preceeding the main stability branch (with $N \geq 63$ ).

These theoretical results correlate again remarkably well with the measured relative abundance spectrum for $\mathrm{Au}_{N}^{3-}$ in the same size range [see Fig. 3(b)]. In particular, note the overall similarity between the trends of $A_{3}$ in Fig. 3(a) and the yields for the trianions in Fig. 3(b), that is, the presence of a weak promontory around $N=55$ in front of the principal branch of the detected $\mathrm{Au}_{N}^{3-}$ clusters. Comparison of the shell-corrected results (solid dots) with the LDM curve (dashed line) further highlights that electronic shell effects underlie the calculated detailed patterns for $A_{3}$ shown in Fig. 3(a) and those observed experimentally in Fig. 3(b).

In addition, our measurements revealed that: (I) Dianionic gold clusters are observed, as expected, for all sizes under investigation $(50 \leq N \leq 70)$, since $n_{a}^{2-}(\mathrm{Au})=12$ [13,16(b)]; and (II) The yields of the stable gold-cluster trianions are two orders of magnitude smaller than the ones corresponding to the parent dianions (which are roughly constant), reflecting the difference in the magnitiudes of the associated multiple electron affinities [see theoretical curves for $A_{2}$ and $A_{3}$ in Fig. 3(a)].

To explore the energetic stability of the $\mathrm{Au}_{N}^{3-}$ clusters against binary $(b)$ and ternary $(t)$ fission [see Eqs. (2) and (3)], we show in Fig. 4 SCM results for the fission dissociation energies, $\Delta_{N, \gamma}^{3-}(\gamma=b$ or $t)$ associated with the most favorable channel for a given parent $\mathrm{Au}_{N}^{3-}$ cluster. We found that the most favorable channel corresponds to the generation of one or two closed-shell $\mathrm{Au}^{-}$ anions in the case of binary and ternary fission, respectively. Thus $\Delta_{N, b}^{3-}=F\left(\mathrm{Au}^{-}\right)+F\left(\mathrm{Au}_{N-1}^{2-}\right)-F\left(\mathrm{Au}_{N}^{3-}\right)$ 
and $\Delta_{N, t}^{3-}=2 F\left(\mathrm{Au}^{-}\right)+F\left(\mathrm{Au}_{N-2}^{-}\right)-F\left(\mathrm{Au}_{N}^{3-}\right)$, with the total free energies of the multiply anionic parent and the charged fission products calculated at $T=300 \mathrm{~K}$. The fission results summarized in Fig. 4 for the most favorable channel illustrate that exothermic fission (that is $\left.\Delta_{N, \gamma}^{3-}<0\right)$ occurs only for the smallest sizes $(N \leq 25)$. This, together with the existence of a fission barrier, leads us to conclude that the decay of $\mathrm{Au}_{N}^{3-}$ clusters is dominated by the electron autodetachment process [see Eq. (画)], which is operative when $A_{3}<0$ and involves tunneling through a CB [16], rather than by fission [26].

The significant findings about the generation, stability patterns, and decay channels, obtained here via Penningtrap experiments and theoretical SCM investigations, include: (i) A remarkable agreement between the theoretically predicted and the measured appearance sizes $\left[n_{a}^{2-}(\mathrm{Ag})=27\right.$ and $\left.n_{a}^{3-}(\mathrm{Au})=54\right]$; (ii) The strong influence of electronic shell effects on the measured yields; and (iii) Identification of electron autodetachment, rather than fragmentation via fission, as the prevalent decay channel (irrespective of the magnitude of the excess negative charge), leading to appearance sizes unrelated to those known from the more familiar case of multiply charged cationic clusters. In light of these findings, it will be of interest to explore further the properties of stored multiplycharged metal cluster anions by either collisional or laser activation [27], as well as investigate their chemical properties 28].

This research is supported by grants from the U.S. Department of Energy (Grant No. FG05-86ER45234), the Deutsche Forschungsgemeinschaft, the EU networks "EUROTRAPS" and "CLUSTER COOLING", the Materials Science Research Center Mainz and the Fonds der Chemischen Industrie.

[1] C. Coulomb, Mémoires de l'Académie, (1786) p. 67ff; ibid. (1787) p. 452; M. Faraday, Experimental research in electricity, (1839) (Dover, New York, 1965), Vol. I, p. $360 f f$.

[2] M.A. Kastner, Physics Today 46, 24 (1993).

[3] R.L. Hettich et al., Phys. Rev. Lett. 67, 1242 (1991); P.A. Limbach et al., J. Am. Chem. Soc. 113, 6795 (1991).

[4] C. Yannouleas and U. Landman, Chem. Phys. Lett. 217, 175 (1994).

[5] X.-B. Wang et al., Phys. Rev. Lett. 81, 3351 (1998).

[6] For experimental studies of cationic clusters, see C. Bréchignac et al., Phys. Rev. Lett. 64, 2893 (1990); ibid. 72, 1636 (1994); Comments At. Mol. Phys. 31, 361 (1995); T.P. Martin et al. Chem. Phys. Lett. 196, 113 (1992); L. Schweikhard et al., Hyperfine Interact. 99, 97 (1996); S. Krückeberg et al., Phys. Rev. A 60, 1251 (1999). U. Näher et al., Phys. Rep. 285, 245 (1997).

[7] R.N. Barnett et al., Phys. Rev. Lett. 67, 3058 (1991); C.
Yannouleas and U. Landman, J. Phys. Chem. 99, 14577 (1995).

[8] For a theoretical review of cationic metal clusters, see C. Yannouleas, U. Landman, and R.N. Barnett in Metal Clusters, edited by W. Ekardt (Wiley, New York, 1999) Ch. 4.

[9] (a) C. Yannouleas and U. Landman, Phys. Rev. B 51, 1902 (1995); (b) Phys. Rev. Lett. 78, 1424 (1997).

[10] J. P. Connerade et al., J. of Phys. B 32, 877 (1999); V. Kasperovich et al., Phys. Rev. Lett. 85, 2729 (2000)

[11] X.-B. Wang and L.-S. Wang, Phys. Rev. Lett. 83, 3402 (1999); P. Weis et al., Chem. Phys. Lett. 321, 426 (2000).

[12] A. Herlert et al., Physica Scripta T80, 200 (1999).

[13] L. Schweikhard et al., Philos. Mag. B 79, 1343 (1999).

[14] A. Herlert et al., Hyperfine Interact. 127, 529 (2000).

[15] C. Stoermer et al. (unpublished).

[16] (a) C. Yannouleas and U. Landman, Chem. Phys. Lett. 210, 437 (1993); Phys. Rev. B 48, 8376 (1993); (b) Phys. Rev. B 61, R10587 (2000).

[17] The relevance of this decay channel for metal clusters was first suggested in the theoretical investigations of Ref. $[16(\mathrm{a})]$, where the appearance sizes of $\mathrm{Na}_{N}^{Z-}, \mathrm{K}_{N}^{Z-}$, and $\mathrm{Al}_{N}^{Z-}(2 \leq Z \leq 4)$ were estimated using the LDM expression (5).

[18] M.A. Preston and R.K. Bhaduri, Structure of the Nucleus (Addison-Wesley, London, 1975).

[19] S. Åberg et al., Phys. Rev. C 58, 3011 (1998).

[20] L. Schweikhard et al., Physica Scripta T59, 236 (1995); L. Schweikhard et al., Eur. Phys. J. D 9, 15 (1999).

[21] T. G. Dietz et al., J. Chem. Phys. 74, 6511 (1981); H. Weidele et al., Z. Phys. D 20, 425 (1991).

[22] H. Schnatz et al., Nucl. Instr. Meth. A251, 17 (1986).

[23] L. S. Brown and G. Gabrielse, Rev. Mod. Phys. 58, 233 (1986); St. Becker et al., Rev. Sci. Instrum. 66, 4902 (1995).

[24] For the $\mathrm{Au}_{N}$ and $\mathrm{Ag}_{N}$ clusters discussed here $(N \leq 80)$, our results at $T=0 \mathrm{~K}$ and $T=300 \mathrm{~K}$ differ only slightly. For cases where the SCM reveals significant thermal effects (i.e., for higher temperatures and/or larger $r_{s}$ 's as in the case of $\mathrm{Na}_{N}$ and $\mathrm{K}_{N}$ clusters), see Ref. [9(b)].

[25] For $\mathrm{Au}_{N}^{Z-}\left(\operatorname{Ag}_{N}^{Z-}\right)$ clusters, the $T=0$ parameters entering in the SCM calculation (for definitions see Ref. $[9(\mathrm{a})]$ ) are $U_{0}=-0.045, r_{s}=3.01$ a.u., $t=0.37(0.47)$ a.u., $\delta_{0}=1.31$ a.u., $\delta_{2}=0, W=5.31(4.26) \mathrm{eV}, \alpha_{v}=-8.06$ $\mathrm{eV}, \alpha_{s}=2.52(2.05) \mathrm{eV}$, and $\alpha_{c}=1.04(0.86) \mathrm{eV}$. For an additional explanation, see footnote 17 in Ref. [9(b)]. The temperature dependence of the surface tension and the coefficient of linear thermal expansion were taken from standard tables.

[26] A similar conclusion can be drawn for the case of doubly anionic metal clusters (see Ref. [16(b)]).

[27] For such studies of singly charged species, see S. Krückeberg et al., J. Chem. Phys. 110, 7216 (1999); U. Hild et al., Phys. Rev. A 57, 2786 (1998); H. Weidele et al., Eur. Phys. J. D 9, 173 (1999); J. Chem. Phys. 110, 8754 (1999).

[28] For the effect of the (negative) charge state (with $Z \leq 1$ ) of gold clusters on their chemical reactivity, see D.M. Cox et al., Z. Phys. D 19, 353 (1991); A. Sanchez et al., J. Phys. Chem. A 103, 9573 (1999). 